\title{
EXPERIMENTAL EVALUATION OF A DIFFERENTIAL GPS-OVER- FIBER SYSTEM FOR AIRCRAFT ATTITUDE DETERMINATION
}

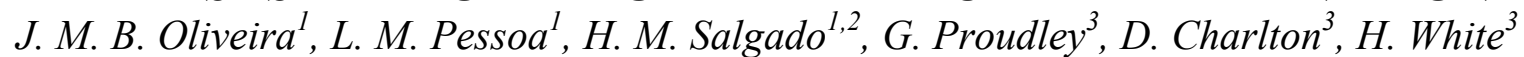 \\ ${ }^{1}$ INESC TEC (formerly INESC Porto) and ${ }^{2}$ Faculty of Engineering, University of Porto, Portugal \\ ${ }^{3}$ BAE Systems Advanced Technology Centre, Bristol, UK
}

\section{Introduction}

The Daphne project has been addressing the adoption of an optical fiber infrastructure for future aircraft [1]. Copper cables are no longer the best option for highly complex systems, particularly when it comes to aerospace systems where size, weight and electromagnetic compatibility are three important requirements. An integrated optical network may be exploited to transport radio frequency (RF) signals from diverse aircraft antennas ranging from satellite/earth communications, collision avoidance, GPS signals for positioning and attitude determination, weather/detection RADAR to corrosion sensors. Aircraft attitude determination using highly precise GPS position systems has been under discussion for several years [2]. The attitude algorithm consists of a highly accurate real time kinematic (RTK) technique, given the short baseline distance between antennas, in which the main antenna acts as a Base station and two auxiliary antennas as Rovers [3]. In [4] we have successfully demonstrated attitude determination by using radio-over-fiber (RoF) techniques to transport carrier-phase (CP) differential GPS signals between the antennas and the respective receivers. In particular, we focused on the performance impact analysis of optical reflections affecting the power level stability of the optical source. This system allows antennas to be placed anywhere in the aircraft jointly with an electro-optic converter while GPS receivers can be centralized several meters away. Here we present a complete functional portable solution for the Remote End (RE) and Head End (HE). Additionally, several results of its performance are also presented and discussed, namely its electrical characteristics and functional behavior in real world scenarios.

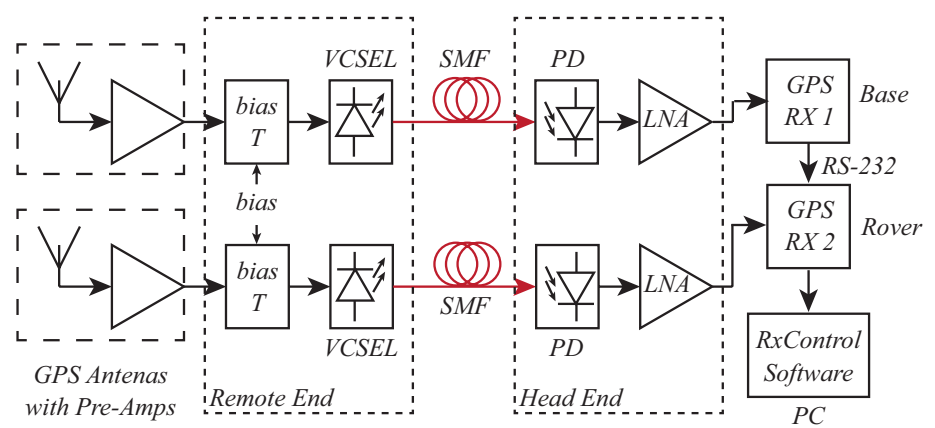

Figure 1. Experimental GPS-over-fiber setup schematic.
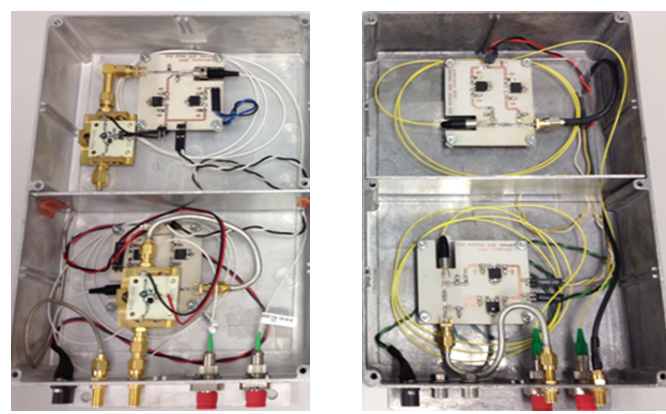

Figure 2. Prototype boxes for (left) Head End and (right) Remote End.

\section{Experimental Setup and Results}

Figure 1 shows the schematic of the experimental setup. A setup consisting of two-antennas (Base and Rover), has been used, which is enough to evaluate the RTK performance. While the electro-optic conversion in the RE is performed by directly modulating RayCan $1550 \mathrm{~nm}$ vertical cavity surface emitting lasers (VCSELs) biased at $6 \mathrm{~mA}$ as in [4], the opto-electronic conversion at the HE is based on AOI photodiodes (PD) suitable for analog applications. Both RE and HE were assembled in RF shielded aluminum boxes (Figure 2). The GPS antennae used were two Septentrio PolaNt dual-frequency with built-in low noise amplification. They were connected to the RE by means of RF cables, and two 150metres single mode fiber (SMF) cables were used to connect the RE to the HE. After the HE, the RF signals were fed into two Septentrio PolaRX GPS receivers (Base and Rover). It should be emphasized that there was no increase in the time to first fix (TTFF) and there was no effect on the GPS position accuracy when the optical link was introduced. Additionally, differences in the optical path lengths did not affect the accuracy of the system since the GPS receivers used were able to cancel out unknown 
delays by resolving double differences between both satellite and receivers signals.

Laboratory measurements were performed using both vector signal and spectrum analyzers. In the band of interest, a magnitude RF response of $0 \mathrm{~dB}$ was obtained and a spurious free dynamic range (SFDR) of $90.7 \mathrm{dBHz}^{2 / 3}$ and a $1 \mathrm{~dB}$ compression point $\left(P_{l d B}\right)$ of $+5 \mathrm{dBm}$ were also measured. Furthermore, the RTK algorithm performance was evaluated when one of the optical paths was subjected to undesirable attenuation. Figure 3 shows the mean TTFF counted from the restart of the algorithm. As it can be seen, a maximum optical attenuation of $11 \mathrm{~dB}$ is achievable for a TTFF around $50 \mathrm{~s}$. Additionally, results for the standard deviation behavior of the latitude, longitude and elevation errors while the GPS is still on fix position are also presented. Finally a cross-talk test was conducted where a Gigabit Ethernet standard signal with 1.25 Gbps directly modulating a VCSEL was multiplexed (at a different wavelength) by means of a coupler in the same Base Station path and used as an interfering signal. Figure 4 shows the TTFF as a function of the optical power of the baseband signal and the corresponding optical isolation necessary at the WDM demux. Also, the standard deviation behavior of the GPS position errors is plotted. As it can be seen, the maximum optical isolation in order to get GPS fix counted from the restart of the algorithm is $12 \mathrm{~dB}$ or, equivalently, the maximum allowed optical power of the baseband signal is $-16 \mathrm{dBm}$. In addition to laboratory tests, the system was also evaluated in a real world scenario using a specially equipped vehicle at BAE Systems facilities. Results showed that the optical system was completely transparent to the GPS signal transmission.

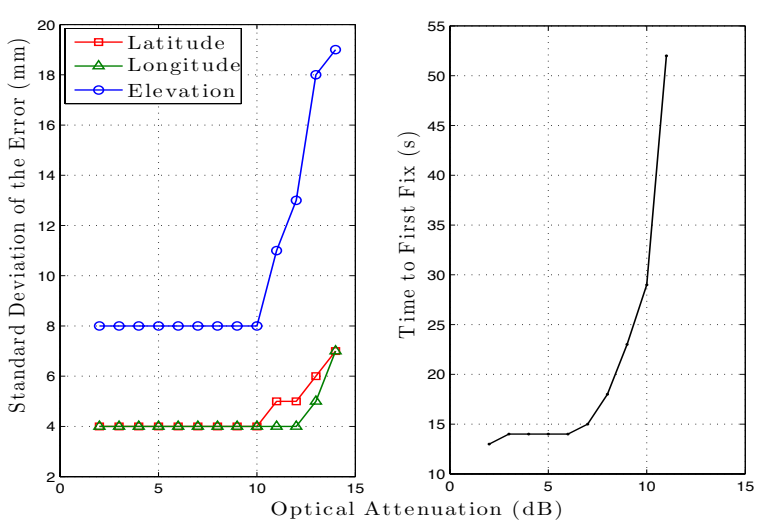

Figure 3. Performance impact in a differential optical attenuation scenario.

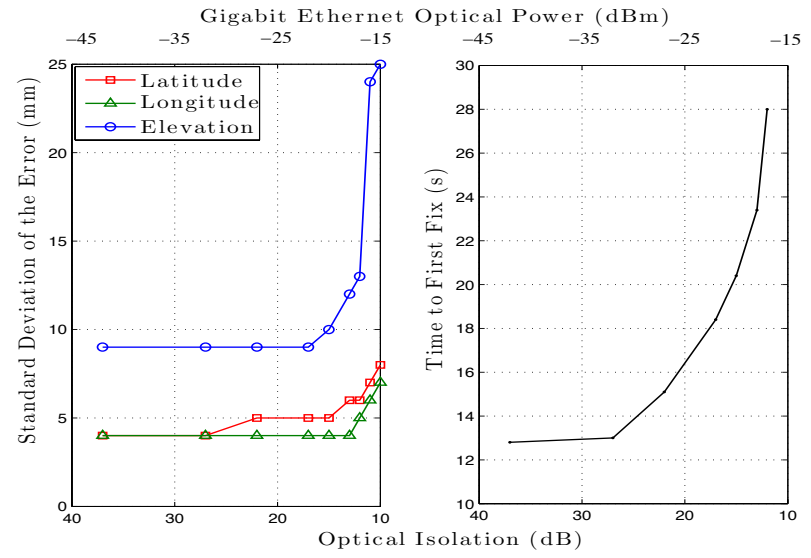

Figure 4. Performance impact in a signal cross-talk scenario.

\section{Conclusion}

We have presented a complete GPS-over-fiber system for aircraft attitude determination. The main conclusions are that in order to a achieve a fix of phase ambiguities in a time slot of $50 \mathrm{~s}$, the maximum optical attenuation of the Base path must be less than $12 \mathrm{~dB}$ and that in a WDM system, the maximum optical power of a baseband signal must be below $-16 \mathrm{dBm}$.

\section{Acknowledgments}

This work is partly funded by FP7 project Daphne, grant ACP8-GA-2009-233709, by the ERDF through COMPETE Programme and by the FCT within project "FCOMP - 01-0124-FEDER-022701" and Programme POCTI/FEDER with grant REEQ/1272/EEI/2005. The authors also acknowledge the support of RayCan.

\section{References}

[1] http://www.fp7daphne.eu, FP7 Project "Developing Aircraft Photonic Networks" website.

[2] Van Graas, F., et al. "GPS Interferometric Attitude and heading determination: Initial flight test results", Navigation, 38, pp. 297-316, Fall 1991.

[3] Simsky A., et al. "Singleboard attitude determination system based on the PolaRx2@GPS receiver", Proceedings of ENC GNSS, 2005.

[4] Pessoa, L. M., et al. "Transmission of differential GPS signals over fiber for aircraft attitude determination", Proceedings of IEEE AVFOP, pp. 80-81, 2012. 